\title{
Effects of Inter-limb Asymmetries on Acceleration and Change of Direction Speed: A Between-Sport Comparison of Professional Soccer and Cricket Athletes
}

\begin{abstract}
The first aim of the present study was to quantify and compare asymmetries among professional soccer and cricket athletes. The second aim was to examine the association between asymmetries and performance within both groups. Professional soccer $(n=18)$ and cricket $(n=23)$ athletes performed single leg countermovement jumps (SLCMJ), single leg drop jumps (SLDJ), a $10 \mathrm{~m}$ sprint and 505 change of direction speed (CODS) tests. Inter-limb asymmetries were calculated as a standard percentage difference, Mann-Whitney $\mathrm{U}$ tests conducted to establish systematic bias between groups and Spearmans $r$ correlations used to establish the relationship between asymmetry scores and speed and CODS performance. Soccer athletes sprinted faster, jumped higher and had a greater reactive strength index (RSI) score than cricket athletes $(p<0.05)$. However, cricketers showed reduced ground contact times compared to footballers during the SLDJ $(p<0.05)$. The cricket group showed significantly greater jump height (asymmetry $=11.49$ vs. $6.51 \% ; p=0.015$ ) and RSI (asymmetry $=10.37$ vs. $5.95 \% ; p=0.014)$ asymmetries compared to soccer players. These metrics were also associated with slower 505 times in the cricket group only ( $r=0.56$ to 0.74 ; $p<0.01)$. These results show that between-limb asymmetries exhibit no association with speed and CODS in elite soccer players, but are associated with reduced CODS in elite cricketers. Thus, the reduction of inter-limb asymmetries may be of greater consideration when working with cricket versus soccer athletes.
\end{abstract}

Key Words: Between-limb differences; elite athletes; physical performance 


\section{INTRODUCTION}

Inter-limb asymmetries have been a common line of investigation in recent years, with numerous studies reporting the prevalence of limb differences across a range of physical testing protocols $(4,21,25,30)$. Such methods include the isometric mid-thigh pull or squat $(11,17)$, isokinetic dynamometry $(9,36)$ and a wide variety of jump tests $(2,4,25,35)$. Previous research has highlighted that asymmetries are a likely consequence of longstanding participation within a single sport (18). However, practitioners must also consider these inter-limb differences within the context of injury risk. The reactive nature of team sports means that equal loading on both limbs is highly unlikely (15). In turn, this may predispose athletes to repetitive loads on the weaker limb that could result in subsequent injury. Regardless of the focus on physical performance or injury risk, their prevalence alone does little to improve our understanding of their impact on measures of physical performance (5). Continuing this line of thought, research to date has shown inconclusive findings $(5,27)$.

Lockie et al. (24) reported between-limb jump height and distance asymmetries in collegiate athletes of 10.4, 5.1 and 3.3\% for the single leg countermovement jump (SLCMJ), single leg lateral and broad jumps respectively. Despite the test-specific asymmetry scores, no association was found with speed or change of direction speed (CODS) in collegiate athletes. Similarly, Dos'Santos et al. (12) used the single leg hop and triple hop for distance tests to report asymmetries of 6.3 and $5.7 \%$. No significant relationships with two CODS tasks were found, also in collegiate athletes. In contrast, Maloney et al. (26) showed that faster recreationally trained adults had significantly smaller jump height asymmetries (2.4 vs. $7.2 \%)$ during the single leg drop jump (SLDJ) when performing a CODS task with multiple $90^{\circ}$ cutting angles. In addition, a significant positive correlation $(r=0.60)$ was reported between jump height asymmetry and CODS times, indicating that larger asymmetries were associated with slower CODS performance. Bishop et al. (4) recently showed that jump height asymmetry from the 
SLCMJ was significantly associated with slower acceleration performance ( $r=0.49$ to 0.59 ) and reduced vertical jump ability $(r=-0.47$ to -0.53$)$ in youth female soccer players. The inconsistency in these findings may be apparent due to discrepancies in the tested populations. With two collegiate samples showing no association $(12,24)$ and arguably two less experienced athlete populations showing an association with reduced performance $(4,26)$, it seems prudent to suggest that strength and training experience may impact the effect that asymmetry has on physical performance (1). Thus, further research examining the effects of inter-limb asymmetries on physical performance is warranted, especially in professional athlete populations.

Given the reactive nature of soccer where players are required to respond to opponents' movement patterns (41), the same volume of workload will likely not be achieved on each limb. In addition, limb dominance will likely change depending on the task in question. For example, players will have a dominant kicking limb, but there is no guarantee that the same limb will be favoured during jumping and cutting actions (13). Thus, the development of asymmetries seems likely in soccer. This is supported in part by previous research from Hart et al. (18) who showed that inter-limb differences in body composition were a by-product of playing team sports over time. Similarly, previous research in cricket has highlighted that abdominal muscle asymmetry is a sport adaptation in fast bowlers, with more symmetrical athletes displaying an increased risk of lower back pain (16). Therefore, between-limb differences in cricket may be a requirement to function optimally in the sport. Given the expected development of inter-limb asymmetries in both soccer and cricket, but huge disparity in how these differences may occur, a direct comparison between the two sports seems warranted. Moreover, understanding whether the effect asymmetry has on physical performance is sport-specific, is key in furthering our understanding as to the relevance of this topic. 
Therefore, the aims of the present study were twofold: 1) to quantify and compare asymmetries among professional soccer and cricket athletes and, 2) to examine the association between asymmetries and performance within both groups.

\section{METHODS}

\section{Experimental Approach to the Problem}

The present study employed the same test battery for both professional cricket (at first class county level) and soccer (from a Tier 3 club in England) athletes. All tests were conducted on the same day (different days for each sport) and took place during the first week of pre-season training. Tests included the SLCMJ, SLDJ, $10 \mathrm{~m}$ sprint and 505 CODS test. Owing to the professional pool of athletes in the present study, test order was not randomized. All athletes completed both jump tests first, followed by the sprint and CODS tests. This order was agreed with the clubs as it was deemed to minimize the accumulation of fatigue and therefore most likely to maximize performances across all tests. In addition, due to the tests being commonly prescribed during strength and conditioning training sessions at each club, test familiarization was deemed sufficient on the day.

\section{Subjects}

Forty-one professional athletes volunteered to participate in this study with the sample split into cricket $(n=23$; age $=20.57 \pm 1.73$ years; height $=1.83 \pm 0.08 \mathrm{~m}$; body mass $=80.23 \pm$ $9.91 \mathrm{~kg})$ and soccer athletes $(n=18$; age $=23.00 \pm 4.27$ years; height $=1.82 \pm 0.06 \mathrm{~m}$; body mass $=78.72 \pm 7.76 \mathrm{~kg}$ ). All athletes were contracted to professional clubs in their respective sports and had a minimum of six years competing at their sport. Both groups had a minimum 
of two years structured strength and conditioning training experience and on average, took part in a minimum of two strength and conditioning sessions per week. Subjects were required to be free from injury for at least two months at the time of testing and deemed fit to participate fully in training and competition by the respective clubs' medical departments. Subjects provided written informed consent and this study was approved by [** DELETED FOR PEER REVIEW **] research and ethics committee.

\section{Procedures}

All testing was conducted at the same time of day (09:00-12:00) to limit the impact of circadian rhythms. Both groups performed a standardized dynamic warm up consisting of multi-planar lunges, inchworms, spidermans and bodyweight squats, followed by three practice trials before each test. Athletes were asked to perform practice trials at 60,80 and $100 \%$ of their perceived maximal effort, with jump tests practiced on both limbs. Three minutes of rest was provided between the last practice trial and the start of the first jump test and 60-seconds of rest was provided between trials during the data collection process.

Single leg countermovement jump. Subjects were instructed to step onto a single uniaxial force platform (PASPORT force plate, PASCO Scientific, California, USA) sampling at $1000 \mathrm{~Hz}$, with their designated test leg and hands placed on hips which were required to remain in the same position for the duration of the test. The jump was initiated by performing a countermovement to a self-selected depth before accelerating vertically as explosively as possible into the air. The test leg was required to remain fully extended throughout the flight phase of the jump before landing back onto the force plate as per the set up. The non-jumping leg was slightly flexed with the foot hovering at mid-shin level, and no additional swinging of 
this leg was allowed during trials. Recorded metrics included jump height, peak propulsive force and concentric impulse, with definitions for their quantification conducted in line with suggestions by Gathercole et al. (14) and Chavda et al. (7) The first meaningful change in force was established when values surpassed five standard deviations (SD) of each participant's body mass (32). Jump height was defined as the maximum height achieved calculated from velocity at take-off multiplied by $2 * 9.81$ (where 9.81 equals gravitational force). Peak propulsive force was defined as the maximum force output during the propulsive phase of the jump and concentric impulse was defined as the net force multiplied by the time taken to produce it; i.e., the area under the force-time curve. The force plate was calibrated prior to each data collection and all force traces were extracted unfiltered, and subsequently copied into a custom-made spreadsheet previously suggested (7). Each athlete performed three trials on each leg with an average score taken on each side to compute the inter-limb asymmetry value. Given the variable nature of inter-limb asymmetry (6), an average asymmetry value was calculated in order to capture some of the variability across both limbs, with the same method employed for the SLDJ test.

Single leg drop jump. The SLDJ was performed using the OptoJump ${ }^{\mathrm{TM}}$ measurement system (Microgate, Bolzano, Italy) with athletes required to step off an $18 \mathrm{~cm}$ box; this height chosen in line with previous research using this test $(25,26)$. With hands fixed on hips, participants were required to step off the box with their designated test leg which subsequently landed on a hard wooden surface between the optimal measurement system below. Upon landing, participants were instructed to minimize ground contact time and jump as high as possible thereafter in line with previous suggestions $(25,26)$. Three jumps were performed on each leg with each side averaged to calculate an inter-limb asymmetry score. Recorded metrics included jump height (calculated from the flight time method), ground contact time (GCT) (time spent 
in contact with the ground after initial landing on the force platform prior to take-off), and reactive strength index (RSI), quantified using the equation flight time/ground contact time (26).

10 m sprint test. Electronic timing gates (Brower Timing Systems, Utah, USA) were positioned at 0 and $10 \mathrm{~m}$ enabling athlete's acceleration ability to be measured. This distance was chosen due to the previously reported importance of acceleration over top speed for team sport athletes $(10,42)$. Athletes started the test in a staggered 2-point stance with toes positioned $30 \mathrm{~cm}$ behind the start line so as to not break the beam of the timing gates prior to the initiation of the test. When ready, subjects sprinted through the timing gates allowing time to be recorded to the nearest hundredth of a second. Three trials were performed in an indoor hall with the fastest trial used for further analysis.

505 change of direction speed test. A distance of $15 \mathrm{~m}$ was measured out with electronic timing gates positioned at the $10 \mathrm{~m}$ mark and the $15 \mathrm{~m}$ point marked out clearly by an existing black line in the hall, to ensure that players had an obvious sight as they approached the turning point. Players sprinted $15 \mathrm{~m}$ and then performed a $180^{\circ}$ turn off both the right and left legs, with a total of three trials completed on each leg. The time started when players broke the electronic beam at the $10 \mathrm{~m}$ mark and after turning $180^{\circ}$, subsequently sprinted back through the timing gates to complete a recorded distance of $10 \mathrm{~m}$. The fastest trial was then used for subsequent data analysis.

\section{Statistical Analysis}


All data were initially recorded as means and SD in Microsoft Excel and later transferred to SPSS (version 24.0; SPSS, Inc., Armonk, NY, USA). All data were checked for normality using the Shapiro-Wilk test and showed non-normal distribution $(p<0.05)$. Within-session reliability of test measures were computed using a two-way random intraclass correlation coefficient (ICC) with absolute agreement and the coefficient of variation (CV). Interpretation of ICC values was in accordance with previous research by Koo and Li, (22) where values > 0.9 = excellent, $0.75-0.9=$ good, $0.5-0.75=$ moderate, and $<0.5=$ poor and CV values were considered acceptable if $<10 \%(8)$.

Mean inter-limb asymmetries were computed using a standard percentage difference equation for both jump tests: $100 /(\max \text { value })^{*}(\min \text { value })^{*}-1+100$, which has been suggested to be accurate for the quantification of asymmetries from unilateral tests $(3,4,6)$ and calculated as an average of three trials on each limb. When depicting inter-limb differences individually, the use of an 'IF function' in Microsoft Excel was added on the end of the formula: $* \operatorname{IF}($ left<right, $1,-1)(6)$, in order to show the direction of asymmetry (i.e., which leg produces the larger score) without altering the magnitude. Spearman's $r$ correlations were conducted to establish the relationship between inter-limb asymmetries and fitness test scores, with statistical significance set at $p<0.05$. Mann-Whitney $\mathrm{U}$ tests were conducted to determine significant differences in mean test scores and inter-limb asymmetry scores between cricket and soccer athletes, with statistical significance set at $p<0.05$. The magnitude of change between sports was quantified using Cohen's $d$ effect sizes (ES): (Mean ${ }_{\text {soccer }}-$ $\left.\mathrm{Mean}_{\text {cricket }}\right) / \mathrm{SD}_{\text {pooled. }}$ These were interpreted in line with suggested scale by Hopkins et al. (20) where $<0.2=$ trivial; $0.2-0.6=$ small; $0.6-1.2=$ moderate; $1.2-2.0=$ large; $2.0-4.0=$ very large; and $>4.0=$ near perfect . 


\section{RESULTS}

Within-session reliability data are presented in Table 1 and shows that all data reported good to excellent reliability, with the exception of GCT on the left limb for cricket athletes (ICC $=$ $0.73)$ and the $10 \mathrm{~m}$ sprint for soccer athletes $(\mathrm{ICC}=0.72)$. When $\mathrm{CV}$ values are considered, all tests showed acceptable variability in both groups $(\leq 9.8 \%)$. Mann-Whitney $\mathrm{U}$ tests revealed that professional soccer athletes showed significantly better performance on the SLDJ test, most notably for jump height on the left leg (22.4 vs. $18.5 \mathrm{~cm} ; p<0.01)$, right leg (22.8 vs. $18.5 \mathrm{~cm} ; p<0.01)$, and $10 \mathrm{~m}$ sprint (1.69 vs. $1.76 \mathrm{~s} ; p<0.01)$.

\section{*** INSERT TABLE 1 ABOUT HERE ***}

Mean inter-limb asymmetry values during the SLCMJ test were (soccer: jump height $=11.14 \%$, peak force $=7.89 \%$, concentric impulse $=8.93 \%$; cricket: jump height $=9.57 \%$, peak force $=$ $8.78 \%$, concentric impulse $=6.39 \%$ ). Mean inter-limb asymmetry values during the SLDJ test were (soccer: jump height $=6.51 \%$, GCT $=6.55 \%$, RSI $=5.95 \%$; cricket: jump height $=$ $11.49 \%, \mathrm{GCT}=6.85 \%, \mathrm{RSI}=10.37 \%)$. Significant differences in asymmetry were shown between sports for jump height $(p=0.015)$ and RSI $(p=0.014)$ during the SLDJ test. No significant differences $(p>0.05)$ in asymmetry were present between groups for the SLCMJ. Individual asymmetry data are presented in Figures 1-4. 
Table 2 shows relationships between inter-limb asymmetry scores and performance during the $10 \mathrm{~m}$ and 505 tests. No significant relationships between asymmetry and performance were present for soccer players in either test ( $r=-0.30$ to 0.39$)$; however, larger asymmetries in jump height $(r=0.56$ to $0.59 ; p<0.01)$ and RSI $(r=0.63$ to $0.74 ; p<0.01)$ during the SLDJ test were indicative of slower times in the 505 test on both limbs for cricket athletes.

\section{*** INSERT TABLE 2 ABOUT HERE ***}

\section{DISCUSSION}

The aims of the present study were to quantify and compare asymmetries among professional soccer and cricket athletes and to examine the association between asymmetries and performance within both groups. Results demonstrated no significant differences in asymmetry between groups for the SLCMJ test. However, significantly larger asymmetries were evident during the SLDJ test in cricket athletes. These SLDJ asymmetries were associated with reduced CODS performance on both limbs in cricket athletes only, but not acceleration (10 m sprint) performance. In addition, soccer players demonstrated significantly faster $10 \mathrm{~m}$ times and jumped higher than cricket athletes during the SLDJ test.

Significant differences were reported between sports during the SLDJ and $10 \mathrm{~m}$ tests (Table 1). Soccer players jumped significantly higher $(E S=0.92$ to 1.10$)$, had a greater RSI score on the right leg $(\mathrm{ES}=0.80)$ and sprinted faster $(\mathrm{ES}=0.93)$ than cricket athletes. This can likely be explained by the typical movement patterns associated with each sport. It has been shown that soccer players can jump between 10-15 times in a game to win headers (29) and perform repeated high intensity actions up to 168 times in a match (40) or on average, every 70 seconds (38). In cricket, comparable data are less common; however, potentially more position-specific. 
As an outfielder, jumping is perhaps likely to occur when reaching for a catch and are likely to be more role-specific. Fast bowlers are likely to employ ballistic jumps prior to ball release during the bowling action (28). Given this repeatable action during bowling, the development of asymmetries could be somewhat expected. When batting, jumping is less common; however, increased loading on one limb is likely given the requirement to move the front limb in to various positions dependent on the shot attempted (39). Thus, the development of asymmetries should also be expected as a consequence of batting and has been reported elsewhere (19). In addition, high intensity sprints are less common (compared to soccer) and have been reported to occur for an average of 1.2 seconds with a work-to-rest ratio of 1:50 (34); thus, representing substantial recovery times (37). Thus, the increased jump performance and faster $10 \mathrm{~m}$ times for soccer athletes in the present study is arguably expected.

In contrast, cricket athletes showed significantly faster GCT's during the SLDJ on the left limb $(E S=0.80)$. That said, there are two possible means of improving an athlete's RSI: jump higher and/or spend less time on the ground. It would appear that soccer athletes' greater jumping ability outweighed cricket athletes' faster GCT's because they also showed significantly greater RSI scores on the right side $(\mathrm{ES}=0.80)$. A definitive explanation for the different jump strategies seen in the present study between sports is challenging. However, given that soccer athletes are likely more familiar with jumping actions compared to cricketers, it seems logical to assume that this may serve as one of the key reasons why significant differences in jump height were seen in a more technically advanced jump, such as the SLDJ.

Figures 1-4 show individual inter-limb asymmetry scores for the SLCMJ and SLDJ tests. The SLCMJ showed no significant differences in asymmetry scores between groups. SLCMJ jump height showed the greatest between-limb asymmetries in both sports $(9.57-11.14 \%)$ and is comparable to previously reported jump height asymmetries during the same test $(4,24)$. Conversely, for the SLDJ, cricket athletes were significantly more asymmetrical than the 
soccer players for jump height $(p=0.015)$ and RSI $(p=0.014)$. This is in part supported by Maloney et al. (26), who showed that jump height asymmetries (also from a SLDJ) were able to distinguish between faster and slower athletes during a CODS test. Given the increased technicality associated with drop jumping compared to the SLCMJ (33), it is possible that this test highlighted larger asymmetries in cricket athletes due to a lack of training at fast stretchshortening-based plyometric activities.

Table 2 shows the correlations between inter-limb asymmetry scores and speed and CODS times. No significant correlations were found in the soccer group in either test; however, significant positive correlations were found between jump height and RSI asymmetries during the SLDJ and 505 times on both limbs in the cricket group. Firstly, it is important to note that these relationships are positive, which indicates that if an asymmetry is larger, the time to perform the 505 test was higher, which is not the desired outcome for any speed or CODS test. Thus, larger drop jump asymmetries are indicative of slower CODS time in professional cricket athletes. The technical nature of the SLDJ test may in part explain these findings. Drop jumps require a very specific transition from braking forces to propulsive forces; this represented by an athlete's ability to spend a short time on the ground and jump as high as possible $(26,33)$. Thus, it seems logical to assume that if substantial asymmetries are present (i.e., $>10 \%)(5,23)$, these may impact performance on a task also governed by effective braking and re-acceleration capacity (31), such as the 505 test. Furthermore, the only significant correlations between asymmetry and performance were in the metrics where cricket athletes showed significantly greater asymmetries than soccer athletes. Thus, the magnitude of asymmetry during the SLDJ would appear to be relevant between groups during the 505 test.

Despite the usefulness of these findings, a few limitations should be acknowledged. Firstly, given the variable and individual nature of asymmetries, these findings are only applicable to the tested soccer and cricket athletes. Further research should aim to establish the effects 
asymmetry has on physical performance comparing across more sporting populations where possible. Furthermore, the methods employed in the present study did not apply a randomized test order. Given the possible effects this may have on fatigue during testing, practitioners are encouraged to adhere to randomized testing. Secondly, inter-limb asymmetries were presented as left vs. right in the present study, rather than defining limbs via dominance. The rationale for this was because different definitions would have been required to define limb dominance, noting that this is a comparison between two different sports. However, practitioners should be mindful that there is no guarantee that the same limb (left or right) will always be the dominant one during athlete populations (13), especially in a sport such as soccer which has distinct positional differences. Thus, defining limbs via dominance may provide practitioners with notable differences in results and are encouraged to perform their own analysis in line with what is deemed most appropriate for their population.

In summary, inter-limb asymmetries measured during the SLDJ test were able to differentiate between professional cricket and soccer athletes. Cricket athletes exhibited significantly greater jump height and RSI asymmetries and these imbalances were also associated with impaired CODS performance during the 505 test on both limbs. Future research should aim to establish if training strategies which target the reduction of inter-limb asymmetries are indicative of improved physical performance.

\section{PRACTICAL APPLICATIONS}

In the present study, larger DJ asymmetries were associated with reduced CODS performance in professional cricket athletes and this was not observed in soccer athletes. Therefore, it seems prudent to suggest that reducing these between-limb differences is warranted within this representative cohort of professional cricketers. Literature has highlighted that unilateral 
training may be favourable over bilateral methods when aiming to minimize asymmetries (14). In this context, given that associations with reduced CODS performance were evident, it is logical to suggest training methods that align with the test goal. Exercises such as unilateral CMJ's, hopping and repeated hurdle hops will encourage a good combination of training for maximal height and true plyometric development. It is also suggested that unilateral strength training not be ignored. Exercises such as split squats, step ups and lunges offer a viable means of targeting each limb independently and should likely be integrated regularly into athlete programs. Therefore, it is reasonable to suggest that such unilateral training methods may address capacity deficits in the weaker limb, which may indirectly improve CODS performance and potentially reduce the risk of injury. 


\section{REFERENCES}

1. Bazyler C, Bailey C, Chiang C-Y, Sato K, and Stone M. The effects of strength training on isometric force production symmetry in recreationally trained males. $J$ Train 3: 610, 2014.

2. Bell D, Sanfilippo J, Binkley N, and Heiderscheit B. Lean mass asymmetry influences force and power asymmetry during jumping in collegiate athletes. J Strength Cond Res 28: 884-891, 2014.

3. Bishop C, Read P, Lake J, Chavda S, \& Turner A. Inter-limb asymmetries: Understanding how to calculate differences from bilateral and unilateral tests. Strength Cond J 40: 1-6, 2018.

4. Bishop C, Read P, McCubbine J, and Turner A. Vertical and horizontal asymmetries are related to slower sprinting and jump performance in elite youth female soccer players. J Strength Cond Res (Published ahead of print).

5. Bishop C, Turner A, and Read P. Effects of inter-limb asymmetries on physical and sports performance: A systematic review. J Sports Sci 36: 1135-1144, 2018.

6. Bishop C, Lake J, Loturco I, Papadopoulos K, Turner A, and Read P. Interlimb asymmetries: The need for an individual approach to data analysis. J Strength Cond Res (Published ahead of print).

7. Chavda S, Bromley T, Jarvis P, Williams S, Bishop C, Turner A, Lake J, and Mundy P. Force-time characteristics of the countermovement jump: Analyzing the curve in Excel. Strength Cond $J$ 2018. (Published ahead of print).

8. Cormack S, Newton R, McGuigan M, and Doyle T. Reliability of measures obtained during single and repeated countermovement jumps. Int J Sports Physiol Perform 3: 131-144, 2008.

9. Costa Silva J, Detanico D, Dal Pupo J, and Freitas C. Bilateral asymmetry of knee and ankle isokinetic torque in soccer players u20 category. Braz J Kinanthro Human Perf 17: 195-204, 2015.

10. Cronin J, and Hansen K. Strength and power predictors of sports speed. J Strength Cond Res 19: 349-357, 2005.

11. Dos'Santos T, Thomas C, Jones P, and Comfort P. Assessing muscle strength asymmetry via a unilateral stance isometric mid-thigh pull. Int J Sports Physiol Perform 12: 505-511, 2017. 
12. Dos'Santos T, Thomas C, Jones P, and Comfort P. Asymmetries in single and triple hop are not detrimental to change of direction speed. J Train 6: 35-41, 2017.

13. Fort-Vanmeerhaeghe A, Gual G, Romero-Rodriguez D and Unnitha V. Lower limb neuromuscular asymmetry in volleyball and basketball players. J Human Kin 50: 135$143,2016$.

14. Gathercole R, Sporer B, Stellingwerff T, and Sleivert G. Alternative countermovementjump analysis to quantify acute neuromuscular fatigue. Int J Sports Physiol Perform 10: 84-92, 2015.

15. Gonzalo-Skok O, Tous-Fajardo J, Suarez-Arrones L, Arjol-Serrano J, Casajus J, and Mendez-Villanueva A. Single-leg power output and between-limbs imbalances in team-sport players: Unilateral versus bilateral combined resistance training. Int J Sports Physiol Perform 12: 106-114, 2017.

16. Gray J, Aginsky KD, Derman W, Vaughan CL, and Hodges PW. Symmetry, not asymmetry, of abdominal muscle morphology is associated with low back pain in cricket fast bowlers. J Sci Med Sport 2016. (Published ahead of print).

17. Hart N, Nimphius S, Spiteri T, and Newton R. Leg strength and lean mass symmetry influences kicking performance in Australian Football. J Sports Sci Med 13: 157-165, 2014.

18. Hart N, Nimphius S, Weber J, Spiteri T, Rantalainen T, Dobbin M, and Newton R. Musculoskeletal asymmetry in football athletes: A product of limb function over time. Med Sci Sports Exerc 48: 1379-1387, 2016.

19. Hides J, Stanton W, Freke M, Wilson S, McMahon S, and Richardson C. MRI study of the size, symmetry and function of the trunk muscles of elite cricketers with and without low back pain. Brit J Sports Med 42: 809-813, 2008.

20. Hopkins W, Marshall S, Batterham A, and Hanin J. Progressive statistics for studies in sports medicine and exercise science. Med Sci Sports Exerc 41: 3-13, 2009.

21. Jones $\mathrm{P}$, and Bampouras T. A comparison of isokinetic and functional methods of assessing bilateral strength imbalance. J Strength Cond Res 24: 1553-1558, 2010.

22. Koo T, and Li M. A guideline of selecting and reporting intraclass correlation coefficients for reliability research. J Chiro Med 15: 155-163, 2016.

23. Kyritsis P, Bahr R, Landreau P, Miladi R, and Witvrouw E. Likelihood of ACL graft rupture: Not meeting six clinical discharge criteria before return to sport is associated with a four times greater risk of rupture. Brit J Sports Med 50: 946-951, 2016. 
24. Lockie R, Callaghan S, Berry S, Cooke E, Jordan C, Luczo T, and Jeffriess M. Relationship between unilateral jumping ability and asymmetry on multidirectional speed in team-sport athletes. J Strength Cond Res 28: 3557-3566, 2014.

25. Maloney S, Fletcher I, and Richards J. A comparison of methods to determine bilateral asymmetries in vertical leg stiffness. J Sports Sci 34: 829-835, 2016.

26. Maloney S, Richards J, Nixon D, Harvey L, and Fletcher I. Do stiffness and asymmetries predict change of direction performance? J Sports Sci 35: 547-556, 2017.

27. Maloney S. The relationship between asymmetry and athletic performance: A critical review. J Strength Cond Res (Published ahead of print).

28. Mukandi I, Turner A, Scott P, and Johnstone J. Strength and conditioning for cricket fast bowlers. Strength Cond J 36: 96-106, 2014.

29. Nedelac M, McCall A, Carling C, Legall F, Berthoin S, and Dupont G. The influence of soccer playing actions on the recovery kinetics after a soccer match. $J$ Strength Cond Res 28: 1517-1523, 2014.

30. Newton R, Gerber A, Nimphius S, Shim J, Doan B, Robertson M, Pearson D, Craig B, Hakkinen K, and Kraemer W. Determination of functional strength imbalance of the lower extremities. J Strength Cond Res 20: 971-977, 2006.

31. Nimphius S, Callaghan S, Spiteri T, and Lockie R. Change of direction deficit: A more isolated measure of change of direction performance than total 505 time. J Strength Cond Res 30: 3024-3032, 2016.

32. Owen N, Watkins J, Kilduff L, Bevan H, and Bennett M. Development of a criterion method to determine peak mechanical power output in a countermovement jump. $J$ Strength Cond Res 28: 1552-1558, 2014.

33. Pedley J, Lloyd R, Read P, Moore I, and Oliver J. Drop jump: A technical model for scientific application. Strength Cond J 39: 36-44, 2017.

34. Petersen C, Pyne D, Dawson B, Kellett A, and Portus M. Movement patterns in cricket vary by both position and game format. J Sports Sci 28: 45-52, 2010.

35. Read P, Oliver J, De Ste Croix M, Myer G, and Lloyd R. The effects of maturation on measures of asymmetry during neuromuscular control tests in elite male youth soccer players. Ped Exerc Sci 30: 168-175, 2017.

36. Ruas C, Brown L, and Pinto R. Lower-extremity side-to-side strength asymmetry of professional soccer players according to playing position. Kinesiology 2: 188-192, 2015. 
37. Scott P, and Herridge R. Chapter 13: Cricket in Routledge Handbook of Strength and Conditioning: Sport-specific Programming for High Performance. Routledge, Abingdon, Oxon, 2018.

38. Stolen T, Chamari K, Castagna C, and Wisloff U. Physiology of soccer: An update. Sports Med 35: 501-536, 2005.

39. Stretch R, Buys F, Du Toit E, and Viljoen G. Kinematics and kinetics of the drive off the front foot in cricket batting. J Sports Sci 16: 711-720, 1998.

40. Taylor J, Wright A, Dischiavi S, Townsend M, and Marmon A. Activity demands during multi-directional team sports: A systematic review. Sports Med 47: 2533-2551, 2017.

41. Turner A, and Stewart P. Strength and conditioning for soccer players. Strength Cond J 36: 1-13, 2014.

42. Young W, James R, and Montgomery I. Is muscle power related to running speed with changes of direction. J Sports Med Phys Fit 42: 282-288, 2002. 
Table 1: Mean test \pm standard deviations (SD), test reliability and effect size data for each sport.

\begin{tabular}{|c|c|c|c|c|c|c|c|}
\hline \multirow{2}{*}{$\begin{array}{c}\text { Fitness } \\
\text { Test }\end{array}$} & \multicolumn{3}{|c|}{ Soccer Athletes $(n=18)$} & \multicolumn{3}{|c|}{ Cricket Athletes $(n=23)$} & \multirow{2}{*}{$\begin{array}{c}\text { Effect size } \\
\text { between sports } \\
(95 \% \mathrm{CI})\end{array}$} \\
\hline & Mean $\pm S D$ & $\begin{array}{l}C V \\
(\%)\end{array}$ & $\begin{array}{c}I C C \\
(95 \% C I)\end{array}$ & Mean $\pm S D$ & $\begin{array}{l}C V \\
(\%)\end{array}$ & $\begin{array}{c}I C C \\
(95 \% C I)\end{array}$ & \\
\hline \multicolumn{8}{|l|}{ SLCMJ: } \\
\hline Jump height-L (m) & $0.18 \pm 0.04$ & 8.18 & $0.95(0.89-0.98)$ & $0.19 \pm 0.03$ & 7.65 & $0.92(0.83-0.96)$ & $0.28(-0.34$ to 0.90$)$ \\
\hline Jump height-R (m) & $0.18 \pm 0.04$ & 8.38 & $0.93(0.85-0.97)$ & $0.18 \pm 0.03$ & 7.85 & $0.94(0.87-0.97)$ & $0.00(-0.62$ to 0.62$)$ \\
\hline Peak force-L $(\mathrm{N})$ & $773.89 \pm 125.47$ & 7.05 & $0.92(0.81-0.97)$ & $761.87 \pm 139.85$ & 9.80 & $0.88(0.76-0.94)$ & $-0.09(-0.71$ to 0.53$)$ \\
\hline Peak force-R $(\mathrm{N})$ & $773.44 \pm 139.52$ & 8.07 & $0.90(0.79-0.96)$ & $726.57 \pm 167.48$ & 8.87 & $0.95(0.89-0.98)$ & $-0.30(-0.92$ to 0.32$)$ \\
\hline CON impulse-L $(\mathrm{N} \cdot \mathrm{s})$ & $131.33 \pm 30.30$ & 7.62 & $0.95(0.87-0.98)$ & $144.43 \pm 25.31$ & 4.49 & $0.98(0.95-0.99)$ & $0.47(-0.16$ to 1.09$)$ \\
\hline CON impulse-R $(\mathrm{N} \cdot \mathrm{s})$ & $135.50 \pm 28.44$ & 7.13 & $0.96(0.91-0.98)$ & $143.00 \pm 25.69$ & 6.23 & $0.95(0.91-0.98)$ & $0.28(-0.34$ to 0.90$)$ \\
\hline \multicolumn{8}{|l|}{ SLDJ: } \\
\hline Jump height-L (m) & $22.39 \pm 4.02 * *$ & 4.94 & $0.97(0.94-0.99)$ & $18.47 \pm 4.45$ & 9.69 & $0.94(0.89-0.97)$ & $-0.92(-1.57$ to -0.28$)$ \\
\hline Jump height-R (m) & $22.82 \pm 3.83 * *$ & 5.55 & $0.93(0.85-0.97)$ & $18.48 \pm 4.06$ & 8.45 & $0.94(0.88-0.97)$ & $-1.10(-1.76$ to -0.44$)$ \\
\hline GCT-L (ms) & $0.29 \pm 0.03 * *$ & 6.34 & $0.88(0.75-0.95)$ & $0.27 \pm 0.02$ & 5.46 & $0.73(0.46-0.88)$ & $-0.78(-1.42$ to -0.14$)$ \\
\hline GCT-R (ms) & $0.29 \pm 0.04$ & 4.55 & $0.92(0.83-0.97)$ & $0.28 \pm 0.02$ & 4.09 & $0.88(0.76-0.94)$ & $-0.32(-0.94$ to 0.30$)$ \\
\hline RSI-L & $1.46 \pm 0.20$ & 6.24 & $0.94(0.87-0.98)$ & $1.37 \pm 0.18$ & 5.11 & $0.94(0.88-0.97)$ & $-0.47(-1.10$ to 0.15$)$ \\
\hline RSI-R & $1.48 \pm 0.21 *$ & 5.01 & $0.94(0.86-0.98)$ & $1.32 \pm 0.19$ & 4.72 & $0.94(0.89-0.97)$ & $-0.80(-1.44$ to -0.16$)$ \\
\hline $10 \mathrm{~m}(\mathrm{~s})$ & $1.69 \pm 0.08 * *$ & 3.83 & $0.72(0.34-0.89)$ & $1.76 \pm 0.07$ & 2.25 & $0.77(0.55-0.90)$ & $0.93(0.28$ to 1.58$)$ \\
\hline $505-\mathrm{L}(\mathrm{s})$ & $2.27 \pm 0.07$ & 1.57 & $0.87(0.65-0.95)$ & $2.21 \pm 0.10$ & 2.25 & $0.86(0.71-0.94)$ & $-0.70(-1.33$ to -0.06$)$ \\
\hline $505-\mathrm{R}(\mathrm{s})$ & $2.26 \pm 0.06$ & 1.24 & $0.85(0.59-0.95)$ & $2.22 \pm 0.12$ & 2.09 & $0.93(0.82-0.97)$ & $-0.42(-1.05$ to 0.20$)$ \\
\hline
\end{tabular}

** indicates significantly different from cricket $(p<0.01)$; * indicates significantly different from cricket $(p<0.05)$

$\mathrm{CV}=$ coefficient of variation; $\mathrm{ICC}=$ intraclass correlation coefficient; $\mathrm{CI}=$ confidence intervals; $\mathrm{SLCMJ}=$ single leg countermovement jump; $\mathrm{CON}=$ concentric; $\mathrm{m}$ = metres; $\mathrm{N}=$ Newtons; $\mathrm{N} \cdot \mathrm{s}=$ Newton seconds; $\mathrm{SLDJ}=$ single leg drop jump; GCT = ground contact time; RSI = reactive strength index; ms $=$ milliseconds; $\mathrm{s}=$ seconds. 


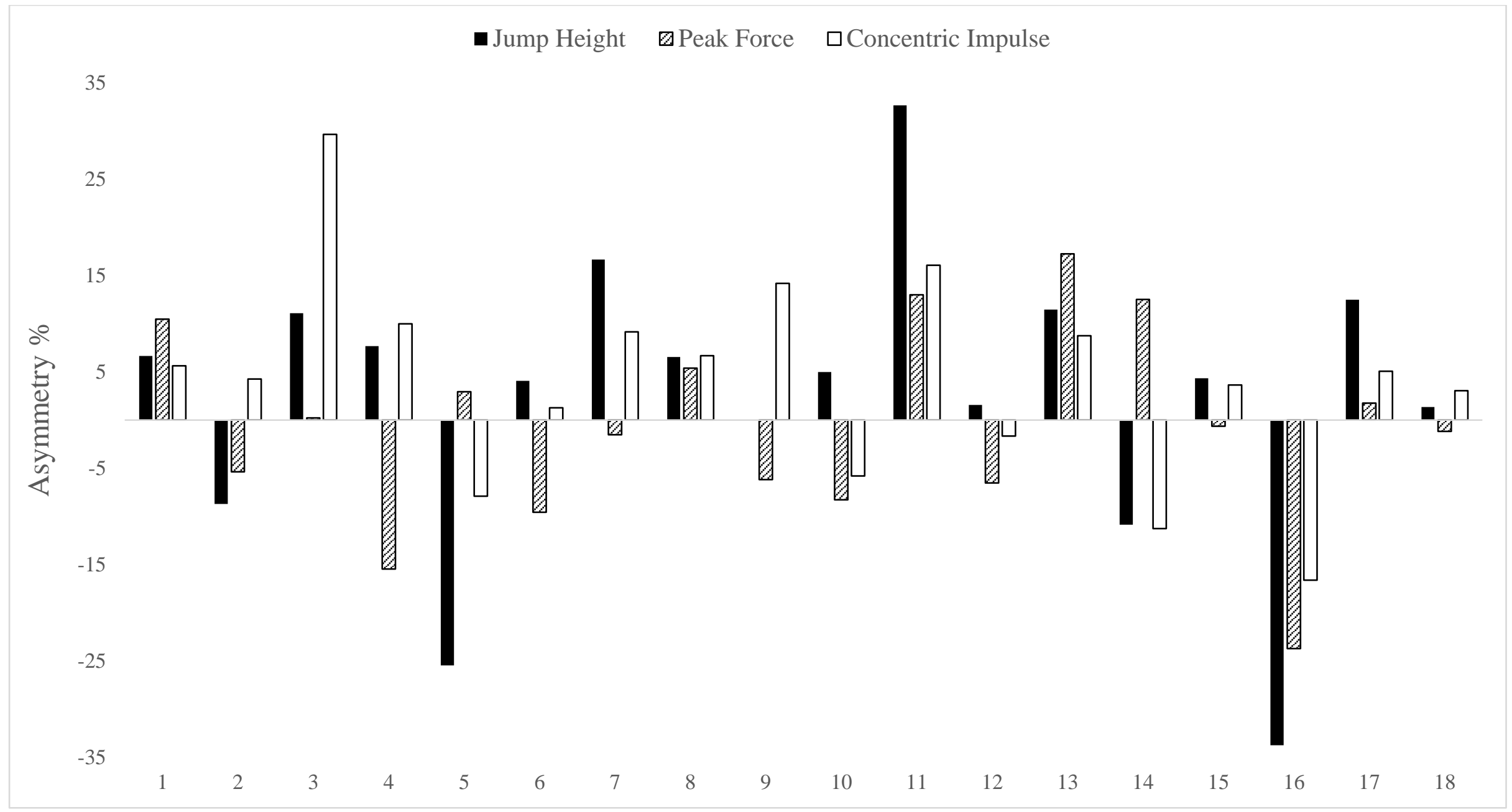

Figure 1: Individual asymmetry data for professional soccer athletes $(n=18)$ during the single leg countermovement jump. Note: above the 0 line indicates asymmetry favours the right leg and below the 0 line asymmetry favours the left leg. 


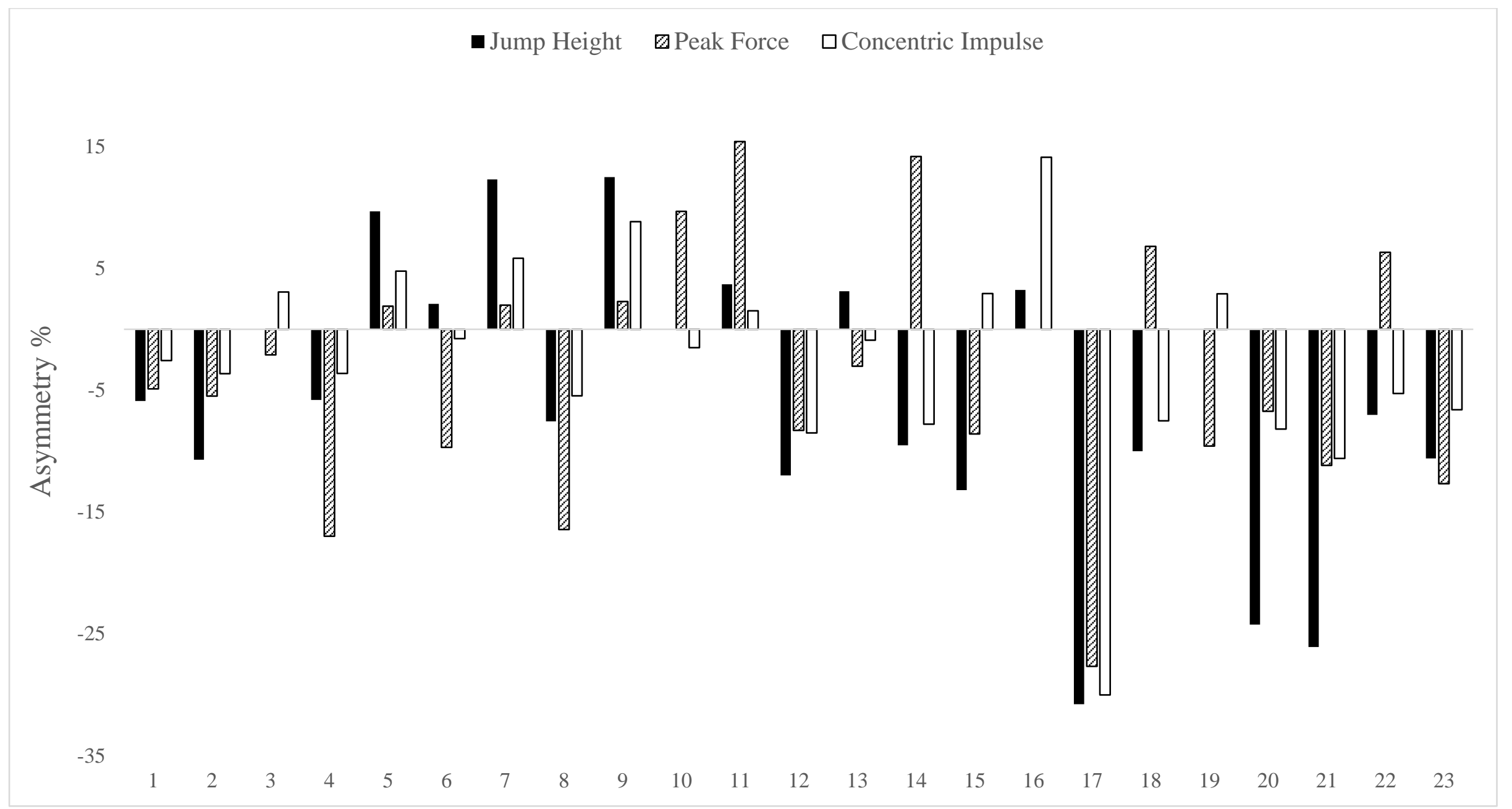

Figure 2: Individual asymmetry data for professional cricket athletes $(n=23)$ during the single leg countermovement jump. Note: above the 0 line indicates asymmetry favours the right leg and below the 0 line asymmetry favours the left leg. 


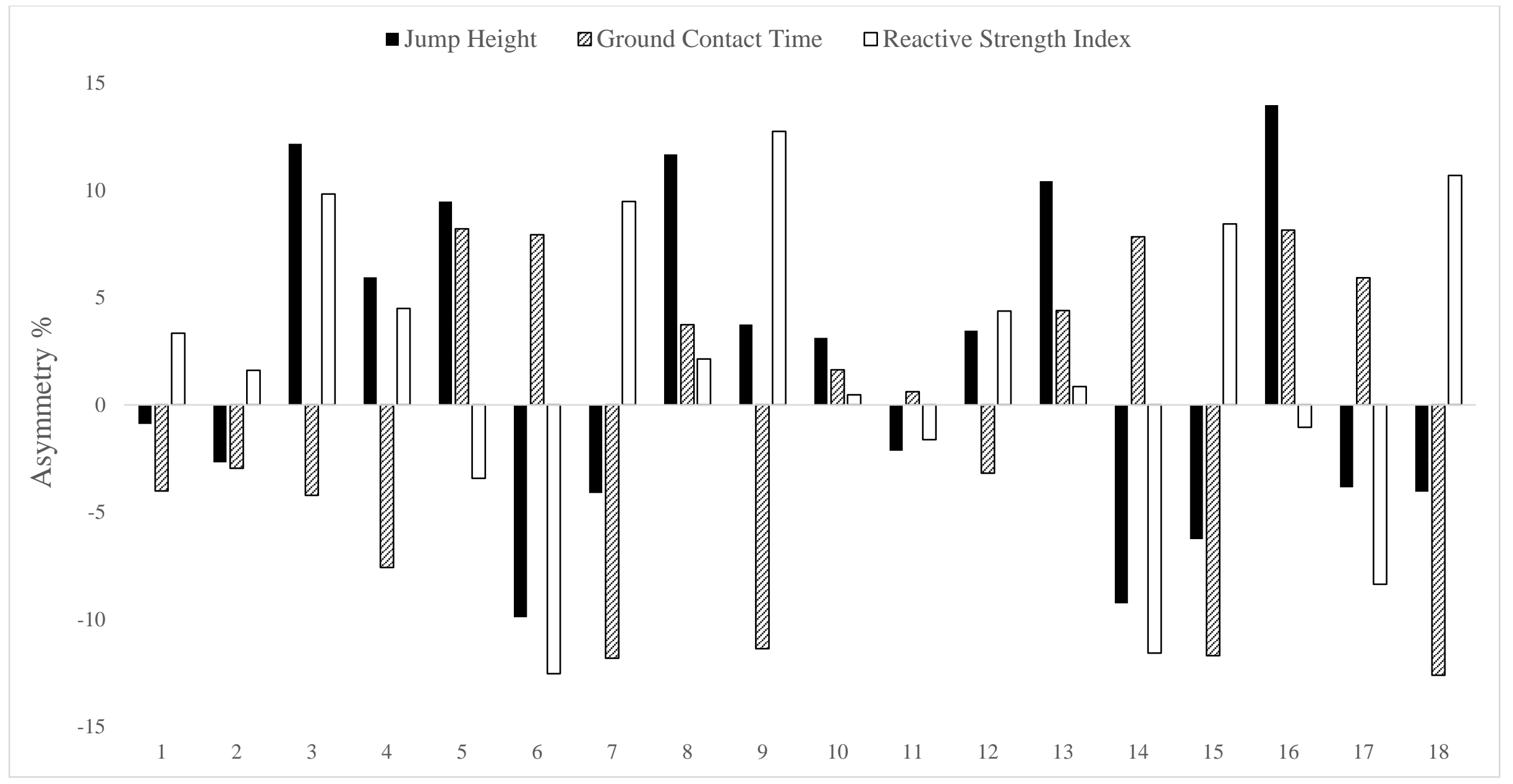

Figure 3: Individual asymmetry data for professional soccer athletes $(n=18)$ during the single leg drop jump. Note: above the 0 line indicates asymmetry favours the right leg and below the 0 line asymmetry favours the left leg. 


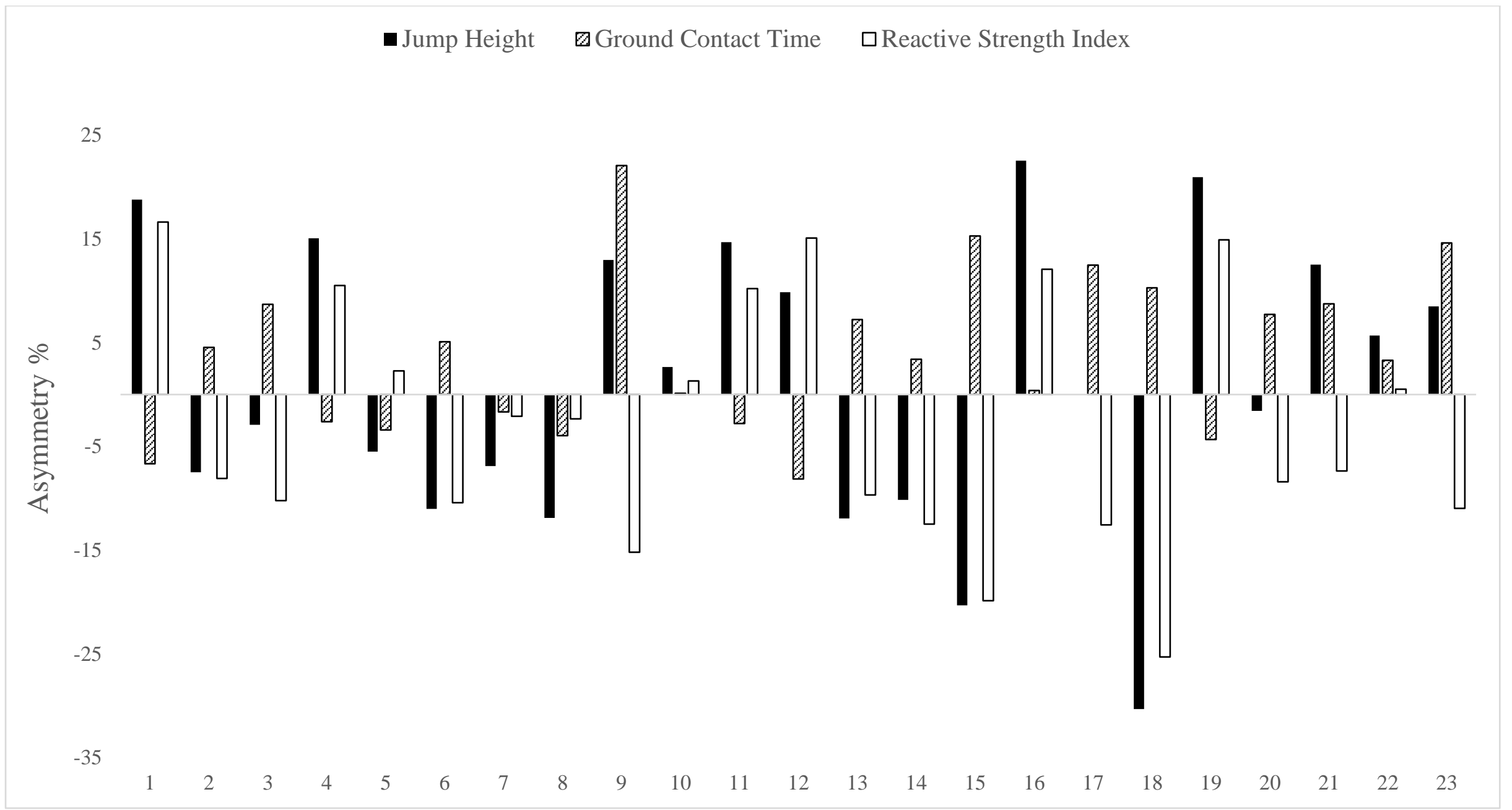

Figure 4: Individual asymmetry data for professional cricket athletes $(n=23)$ during the single leg drop jump. Note: above the 0 line indicates asymmetry favours the right leg and below the 0 line asymmetry favours the left leg. 
Table 2: Spearman's $r$ correlations (95\% confidence intervals) between inter-limb asymmetry scores and speed and change of direction speed tests.

\begin{tabular}{|c|c|c|c|c|c|c|}
\hline \multirow{2}{*}{$\begin{array}{c}\text { Asymmetry } \\
\text { Test/Metric (\%) }\end{array}$} & \multicolumn{3}{|c|}{ Soccer Athletes $(n=18)$} & \multicolumn{3}{|c|}{ Cricket Athletes $(n=23)$} \\
\hline & $10 \mathrm{~m}$ & $505($ left $)$ & 505 (right) & $10 \mathrm{~m}$ & $505($ left $)$ & 505 (right) \\
\hline \multicolumn{7}{|l|}{ SLCMJ: } \\
\hline Jump height & $0.11(-0.38$ to 0.55$)$ & $-0.23(-0.63$ to 0.27$)$ & $0.14(-0.35$ to 0.57$)$ & $-0.07(-0.52$ to 0.41$)$ & $0.03(-0.44$ to 0.49$)$ & $0.07(-0.41$ to 0.52$)$ \\
\hline Peak force & $-0.30(-0.67$ to 0.19$)$ & $-0.07(-0.52$ to 0.41$)$ & $-0.03(-0.49$ to 0.44$)$ & $-0.07(-0.52$ to 0.41$)$ & $0.22(-0.28$ to 0.62$)$ & $0.15(-0.34$ to 0.58$)$ \\
\hline CON impulse & $0.33(-0.16$ to 0.69$)$ & $-0.23(-0.63$ to 0.27$)$ & $0.29(-0.21$ to 0.67$)$ & $0.36(-0.13$ to 0.71$)$ & $0.37(-0.12$ to 0.71$)$ & $0.27(-0.23$ to 0.65$)$ \\
\hline \multicolumn{7}{|l|}{$S L D J:$} \\
\hline Jump height & $0.18(-0.31$ to 0.60$)$ & $0.39(-0.09$ to 0.73$)$ & $0.29(-0.21$ to 0.67$)$ & $0.28(-0.22$ to 0.66$)$ & $0.56(0.13 \text { to } 0.81)^{*}$ & $0.59(0.17 \text { to } 0.83)^{*}$ \\
\hline GCT & $0.20(-0.29$ to 0.61$)$ & $0.15(-0.34$ to 0.58$)$ & $-0.12(-0.56$ to 0.37$)$ & $0.02(-0.45$ to 0.48$)$ & $0.23(-0.27$ to 0.63$)$ & $0.10(-0.39$ to 0.54$)$ \\
\hline RSI & $0.35(-0.14$ to 0.70$)$ & $0.23(-0.27$ to 0.63$)$ & $-0.12(-0.56$ to 0.37$)$ & $0.36(-0.13$ to 0.71$)$ & $0.74(0.42 \text { to } 0.90)^{*}$ & $0.63(0.23 \text { to } 0.85)^{*}$ \\
\hline
\end{tabular}

* indicates statistical significance at $p<0.01$

SLCMJ = single leg countermovement jump; CON = concentric $;$ SLDJ = single leg drop jump; GCT = ground contact time; $\mathrm{RSI}=$ reactive strength index . 